\section{PULSELESS ELECTRICAL ACTIVITY}

Deandra Luong and colleagues report a series of infants where ECG monitoring was used to monitor the heart rate during initial stabilisation after birth and there was no clinically detectable cardiac output despite ECG traces showing heart rates of up to 90 beats per minute. These cases of cardiac arrest with pulseless electrical activity (PEA) rhythm in newborn infants may be more common than previously recognised because immediate ECG recording during neonatal resuscitation has not been in common usage. A study from the same group in asphyxiated piglets with asystole showed PEA in 43\%. Although ECG may provide a measure of heart rate more rapidly than a saturation monitor, it should not be relied on in isolation to monitor the response to resuscitation. Initial and ongoing periodic clinical assessment of the circulation by auscultation of the heart rate and palpation of the pulses is still required. See page F572.

\section{DELAYED CORD CLAMPING IN MULTIPLES}

Now that there is clear evidence that delayed cord clamping (DCC) in preterm infants significantly reduces their risk of mortality, any decision that this is contra-indicated requires careful justification. Exclusion should ideally be on the basis of evidence of actual risk rather than hypothetical risk because there is now proven risk from immediate cord clamping. Priya Jegatheesan and colleagues report retrospective observational data on 529 preterm infants who had DCC of at least $30 \mathrm{~s}$. Their series included 29 nine pairs of monochorionic twins. In 22 of these pairs both twins received DCC and in a further five pairs, one of the twins did so. None of these infants had polycythaemia. One had anaemia. This is a group of infants commonly excluded from DCC by others. Further data describing the outcomes of DCC in monochorionic twins would be of value as these infants are not few in number and they may be missing out on an opportunity to benefit from the survival advantage of DCC on the basis of a hypothetical risk that is in reality quite rare. See page F575.

\section{LESS INVASIVE SURFACTANT ADMINISTRATION}

Egbert Herting and colleagues provide a comprehensive review on the use of less invasive surfactant administration (LISA) techniques. Individual studies and meta-analyses that are reviewed point in the direction that LISA is more effective than standard treatment or the INtubate-SURfactant-Extubate (INSURE) method of surfactant administration both in terms of short-term (avoidance of mechanical ventilation) and long-term (intracerebral haemorrhage and bronchopulmonary dysplasia) outcomes. LISA is not presented as an isolated procedure, but more as a part of a wider care bundle aimed at optimising adaptation to extrauterine life under spontaneous breathing. In a separate report, Lobke Janssen and colleagues describe factors associated with failure of the broadly similar Minimally Invasive Surfactant Treatment (MIST). Failure was defined as need for ventilation in the next 72 hours. Among 185 preterm infants treated in two centres in The Netherlands, initial surfactant dose $<200 \mathrm{mg}$ / $\mathrm{kg}$ was a modifiable post-natal factor associated with treatment failure. See pages F655 and F636

\section{RESUSCITATION TRAINING-TRIAL OF A RESPIRATORY FUNCTION MONITOR}

In a randomised trial that included 400 participants, Eoin O'Currain and colleagues showed that when training was supported by information from a respiratory function monitor, trainees achieved lower mask leak and better delivered tidal volumes in simulated neonatal resuscitation scenarios. Effective mask ventilation of newborn infants is such a vital skill that this objective demonstration that this training intervention is effective and feasible should influence training more widely. See page F582.

\section{FENTANYL CLEARANCE}

In a pharmacokinetic study performed in 98 preterm infants born before 32 weeks gestation, Swantje Völler and colleagues observed wide variation in drug clearance that was related to both gestational age and postnatal age. There may be appreciable risk of drug accumulation in lower gestational age infants in the early days after birth. See page 598.

\section{ONE-YEAR OUTCOMES OF SURGERY FOR CONGENITAL DIAPHRAGMATIC HERNIA}

Anna-May Long and colleagues report a prospective national population cohort study of liveborn infants with congenital diaphragmatic hernia. The study took place in paediatric surgical centres in the UK and Ireland over an 18 month period and included 219 infants. Overall survival to 1 year was $75 \%$ and post-operative survival was $93 \%$. Patch repair was associated with a high risk of post-operative chylothorax, particularly when the patch material was synthetic. See page F643. 